\title{
Effet du degré de maturation sur la cinétique de fermentation éthylique de la pulpe de mangue cultivar BOKO
}

\author{
Philippe Diakabana ${ }^{1}{ }^{\star}$, Simon C. Kobawila², V. Massengo ${ }^{2}$, Delphin Louembé2.
}

${ }^{1}$ Centre de Recherche et d'Initiation des Projets de Technologie, Délégation Générale à la Recherche Scientifique et Technologique, BP : 2499, Brazzaville, Congo

2EPRAN-Congo, Faculté des Sciences, Université Marien NGOUABI, BP : 69, Brazzaville, Congo

*Auteur correspondant, E-mail : diakabanap@yahoo.fr

\begin{abstract}
RESUME
Le fruit de la mangue "Boko » présente des caractéristiques physiques et organoleptiques très particulières comparées aux autres mangues produites au Congo. La masse de ce fruit est de $390 \mathrm{~g}$ en moyenne et très proche de celle des variétés lippens et broagt, mais son rendement en pulpe (67\% de la masse du fruit) est nettement plus élevé. Sa largeur $(107,94 \pm 20,09 \mathrm{~mm})$ et son épaisseur $(72,27 \pm 5,35 \mathrm{~mm})$ sont assez régulières mais son indice de sphéricité $(0,88 \pm 0,52)$ est très dispersé. Les diverses caractéristiques physicochimiques du fruit de la mangue "Boko" sont fonction du degré de maturation physiologique évalué à partir de l'indice de maturation $\beta$ (de $\beta_{1}=18,22$ à $\left.\beta_{3}=83,5\right)$. La surmaturation de ce fruit intervient lorsque la valeur de l'indice de maturation est supérieur $\left(\beta_{3}=83,5\right)$. Elle correspond à une forte diminution du degré d'acidité de $72,6 \%$, une diminution significative de la teneur en eau (de $83,98 \%$ à $81,18 \%$ d'eau), mais à une augmentation considérable de la teneur en sucre (de 10 à $19^{\circ}$ Brix). La faible acidité ( $\left.2 \mathrm{~g} \mathrm{de} \mathrm{H}_{2} \mathrm{SO}_{4} / \mathrm{l}\right)$ de la pulpe de mangue surmaturée est responsable de la fermentation incomplète du jus testé. Afin d'améliorer la productivité horaire en éthanol du jus de pulpe de mangue surmaturée, il serait nécessaire de procéder à une acidification indirecte par l'apport d'une portion de pulpe de mangue moins mûre.
\end{abstract}

Mots clés : Morphologie du fruit ; mangue "Boko" ; indice de maturation ; productivité en éthanol.

\section{ABSTRACT}

Effect of the maturation index on the kinetic of ethyl fermentation of juice from the mango cultivar"Boko" pulp.

The fruit of the mango"boko" presents physical and organoleptic characteristics very special compared to other mangoes produced in Congo. The mass of this fruit is $390 \mathrm{~g}$ on average and very close to those of lippens and broagt varieties, but its pulp yield $(67 \%$ of the mass of fruit) is much higher. Its width $(107,94 \pm 20,09 \mathrm{~mm})$ and thickness $(72,27 \pm 5,35 \mathrm{~mm})$ are fairly regular, but the sphericity index $(0,88 \pm 0,52)$ is very varied. The various physicochemical characteristics of mango "boko" fruit depend on the degree of physiological maturity assessed from the maturation index $\beta$ (from $\beta_{1}=18.22$ to $\beta_{3}=83.5$ ). The over-ripening of this fruit occurs when the value of the maturation index was higher $\left(\beta_{3}=83.5\right)$ and corresponds to a decrease pronounced of degree of acidity of $72.6 \%$, a significant decrease in water content (from $83.98 \%$ to $81.18 \%$ of water), but a considerable increase in sugar content (from $10^{\circ}$ Brix to $19^{\circ}$ Brix). The low acidity $\left(2 \mathrm{~g} \mathrm{of} \mathrm{H}_{2} \mathrm{SO}_{4} / \mathrm{ll}\right.$ ) of overripened mango pulp is responsible for the incomplete fermentation of juice tested. To improve the hourly productivity in ethanol from juice of ripened mango pulp, it would be necessary to acidify indirectly by providing a portion of mango pulp less mature.

Key words: Fruit morphology, mango"boko", maturation index; ethanol productivity.

\section{INTRODUCTION}

Le manguier (Mangifera indica L.) est cultivé dans les tropiques pour ses fruits utilisés dans la préparation de condiments, desserts, sorbets et boissons [1, 2]. En raison de la composition chimique de la pulpe, la consommation de la mangue est recommandée dans les cas d'affections de la peau et de la rétine, d'artériosclérose, de l'hypertension artérielle et du diabète [3]. Cela montre l'intérêt de la vulgarisation alimentaire de la pulpe de mangue.
Juteuse et parfumée, la pulpe de la mangue permet la préparation, en Afrique, de nombreux produits transformés de la mangue. A l'échelle artisanale, le jus de mangue est transformé par fermentation éthylique spontanée ou contrôlée [4]. Comme produits de transformation industrielle dans d'autres pays [2], on peut citer les tranches de pulpe de mangue au sirop ou séchées [5] et les semi-confits. Au Congo, les stations fruitières des localités de Boko et de Loudima, situées respectivement dans les Départements du Pool et 
de la Bouenza, s'occupent de la vulgarisation de certaines variétés de mangues. A la station fruitière de Boko, une variété de mangue améliorée, mal connue et localement appelée mangue "Boko", est très appréciée pour ses qualités gustatives et aromatiques.

Dans le but de valoriser la mangue "Boko", le fruit et la pulpe ont été caractérisés, et la fermentation du jus de la pulpe étudiée en fonction du degré de maturation du fruit.

\section{MATERIEL ET METHODES}

\section{Echantillonnage}

Les fruits de la mangue "Boko", ont été prélevés sur trois pieds de manguier, ensuite homogénéisés et répartis en trois lots de $20 \mathrm{~kg}$, sur la base de la couleur de la peau (jaune-vert, jaune clair et jaune foncé) et de la teneur en sucres totaux ( ${ }^{\circ}$ Brix). Chacun des lots correspond respectivement à la gamme des fruits légèrement mûrs ou lot I $\left(10^{\circ}\right.$ Brix- $\left.13,5^{\circ} \mathrm{Brix}\right)$, des fruits parfaitement mûrs ou lot $\|\left(14^{\circ}\right.$ Brix- $15,5^{\circ}$ Brix $)$ et des fruits surmaturés ou lot III (16 $\left.\mathrm{Brix}-18^{\circ} \mathrm{Brix}\right)$.

2. Caractérisation morphologique et physique des fruits

Les grandeurs géométriques considérées dans l'appréciation de la morphologie du fruit de la mangue "Boko" ont été les suivantes (Photo 1):

a) longueur $a$, largeur $b$ et épaisseur $c$ du fruit ;

b) indice de calibre $I_{c}=\sqrt{a \cdot b \cdot c}$ et indice de sphéricité $I s=\sqrt[z]{a \cdot b \cdot c_{/ a}}[6]$. La mesure de dimensions a été effectuée au moyen d'un pied à coulisse.

La masse totale du fruit a été mesurée au moyen d'une balance de précision $0,01 \mathrm{~g}$. Après avoir ouvert longitudinalement le fruit à l'aide d'un couteau, la graine et la peau ou écorce ont été séparées de la pulpe; les mesures de la masse des composantes du fruit ont été déterminées par pesée.

La proportion en pulpe $\left(P_{p}\right)$ a été déterminée par le rapport de la masse totale de la pulpe sur la masse totale des 120 fruits et exprimée en pourcentage [5].

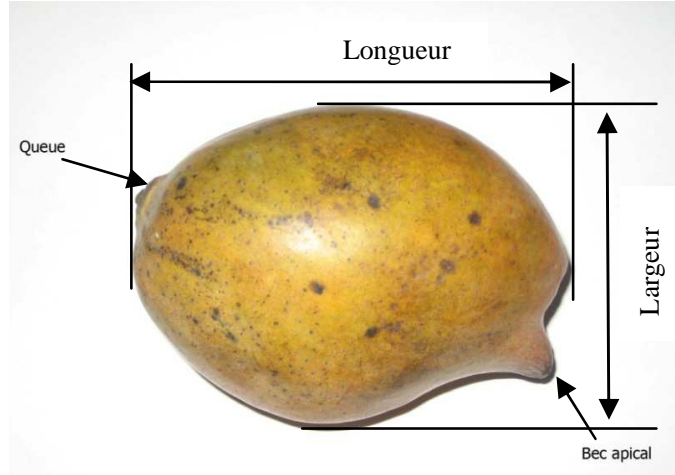

Photo 1: Fruit de la mangue Boko: Longueur, largeur, épaisseur= hauteur suivant l'axe vertical au cœur du produit.

Le volume de la mangue a été apprécié en introduisant le fruit entier dans une éprouvette graduée contenant de l'eau de volume connu. La différence de volume avant et après immersion totale du fruit correspond au volume de la mangue $\left(V_{z}\right)$.

La masse volumique $\rho$ du fruit a été évaluée par le rapport de la masse du fruit $\left(M_{t}\right)$ sur son volume $\left(V_{t}\right)$ selon la formule suivante : $\rho=M_{t / V_{t}}\left(\mathrm{~g} / \mathrm{cm}^{3}\right)$

3. Caractérisation physico-chimique de la pulpe Les teneurs en eau et en cendres de la pulpe de mangue ont été déterminées selon les méthodes d'analyse basées sur la dessiccation, décrites dans les Annexes des Directives de la C.E.E. [7]. La teneur en sucres totaux de la pulpe de mangue a été déterminée par réfractométrie [8] en degré Brix.

L'acidité totale a été mesurée par titrimétrie [9; 10]. Elle est exprimée en grammes de $\mathrm{H}_{2} \mathrm{SO}_{4} / \mathrm{l}$ selon la formule suivante :

Acidité totale $=0,49 \mathrm{~g} \times n \mathrm{ml}$ de solution $\mathrm{NaOH} 0,1 \mathrm{~N}$ utilisée ( $g$ de $\left.\mathrm{H}_{2} \mathrm{SO}_{4} / \mathrm{l}\right)$.

En vue de mettre en évidence le profil de saccharification physiologique de la pulpe de mangue suivant le degré de maturation du fruit, le test à l'iode $0,05 \mathrm{~N}$ [11] a été réalisé pour visualiser l'intensité de la présence d'amidon.

Le degré de maturation physiologique du fruit de la mangue "Boko" a été apprécié à partir de l'indice de maturation $\beta$ d'un échantillon de jus de mangue considéré $[2 ; 8]$ obtenu après 
pressurage complet de la pulpe et homogénéisation du jus de l'ensemble de l'échantillon. Après avoir mesuré la teneur en sucres par pycnométrie [10] et l'acidité totale par titrimétrie, l'indice de maturation $\beta$ a été déterminé selon la formule suivante :

$$
\beta=S / A
$$

avec $S=$ teneur en sucres $(g / l)$ et

$A=$ acidité totale $\left(g\right.$ de $\mathrm{H}_{2} \mathrm{SO}_{4} /$ ).

4. Extraction du jus de mangue "Boko" Après épluchage et dépulpage du fruit à l'aide d'un couteau, la pulpe obtenue a été finement broyée au moyen d'un broyeur à stries. La purée obtenue a été traitée différemment suivant deux voies d'utilisation :

a) pour l'analyse du jus de mangue, la purée, placée sur une toile de mousseline, a été pressée manuellement par torsade et le jus trouble obtenu a été filtré au moyen d'un papier-filtre ;

b) pour le jus destiné à la fermentation éthylique contrôlée, la masse de la purée a été préalablement diluée avec de l'eau $(20 \% \mathrm{w} / \mathrm{v})$. Après pressage de la pulpe diluée et filtration du jus trouble, le jus clair obtenu a été standardisé par sucrage à sec à $20 \%$ de sucre, ensuite pasteurisé.

\section{Fermentation du jus de pulpe de mangue Préparation de l'inoculum}

Six (6) grammes de levure sèche Saccharomyces bayanus CH158/SIHA ont été réactivés par réhydratation dans un bécher de $100 \mathrm{ml}$ contenant $44 \mathrm{ml}$ d'une solution de saccharose à $12 \%$ (w/w). Le mélange a été ensuite homogénéisé et incubé à $27^{\circ} \mathrm{C}$ pendant 90 minutes [12]. On a obtenu ainsi un inoculum.

Préparation du moût

Le moût a été standardisé à $20^{\circ}$ Brix par addition de sucre de canne sur du jus de pulpe de mangue puis pasteurisé à $85^{\circ} \mathrm{C}$ pendant 20 minutes pour détruire la flore endogène $[4 ; 13]$.

Le moût standardisé, pasteurisé et refroidi à $27^{\circ} \mathrm{C}$, a été ensuite enrichi en sulfate d'ammonium $(0.143 \mathrm{~g} / \mathrm{l})$ et extrait de levure $(0.285 \mathrm{~g} / \mathrm{l})[14 ; 15]$.

Fermentation éthylique

La fermentation contrôlée a été faite avec le moût inoculé avec la préparation réactivée de souche de levure Saccharomyces bayanus CH158/ SIHA dans le ratio inoculum/moût=1/500 (V/V).

Dosage des sucres totaux et de l'éthanol
La teneur en sucres totaux du moût a été déterminée par pycnometrie en $\%$ poids [16] et réfractométrie en degré Brix [8].

Le dosage d'éthanol du moût en fermentation a été fait par la méthode pycnométrique EBC en $\%$ poids [17].

Rendement de la transformation des sucres en éthanol et productivité en éthanol

Le rendement de transformation de sucre Rt [18; 19] a été évalué à partir de la relation suivante adaptée par Diakabana [20]:

$$
R t=s_{t} / s_{0} \times 100
$$

avec : $S t$ = quantité de sucre transformé ;

$\mathrm{Sc}=$ quantité de sucre consommé $(\mathrm{g} / \mathrm{l})$;

So= quantité initiale de sucre $(\mathrm{g} / \mathrm{l})$;

$S r=$ quantité de sucre résiduel $(g / l)$ et $S t=S c=S o-S r$.

Le rendement de conversion de sucre en éthanol Rc a été déterminé selon la formule suivante [18 ; 19] adaptée par Diakabana [20] :

$$
\mathrm{Rc}=E_{t} / s_{e} \times 100
$$

avec : Et = teneur en éthanol formé et $\mathrm{Sc}=$ teneur en sucre consommé $(\mathrm{g} / \mathrm{l})$.

La teneur en éthanol a été retenue comme critère de performance [19] pour évaluer les différents essais de fermentation testés en tenant compte du temps, une des contraintes économiques. La productivité totale horaire en éthanol $\mathrm{P}_{\mathrm{TE}}$ a été déterminée selon la relation suivante adaptée par Diakabana [20] :

$$
\boldsymbol{P}_{T E}=\frac{E_{f}}{t_{M}}
$$

avec $E t$ : teneur final en éthanol $(g / l)=$ teneur maximale en éthanol évaluée en fin de fermentation;

$T m$ : durée totale d'un cycle de fermentation (en heure) et

$P_{T E}$ (en g d'éthanol/kg de pulpe par heure).

Au cours de la fermentation, la valeur maximale d'éthanol ou teneur finale en éthanol $E_{f}$ a été obtenue à un temps maximal tm ou temps limite correspondant à une durée totale ou durée finale du processus de fermentation.

\section{Analyse statistique}

Dans le cadre de la caractérisation du fruit de la mangue "Boko" et l'étude cinétique de la fermentation éthylique de jus de mangue extrait 
respectivement de trois lots de 40 fruits (mangues légèrement maturées, maturées et surmaturées), les valeurs statistiques suivantes ont été considérées : moyenne, écart-type et intervalle de confiance [moyenne \pm écart-type]. La méthode statistique modifiée basée sur la loi en cloche de Gauss-Laplace a été utilisée [21].

\section{RESULTATS}

1. Caractérisation morphologique du fruit de la mangue "Boko"

Le fruit de la mangue "Boko" est particulièrement remarquable par sa terminaison apicale en forme de bec (Photo 1). Les résultats sur les caractéristiques morphologiques (Tableau 1) révèlent que ce fruit a une longueur de
$107,94 \pm 20,09 \mathrm{~mm}$, une largeur de $84,25 \pm 6,36$ $\mathrm{mm}$ et une épaisseur de $72,27 \pm 5,35 \mathrm{~mm}$. Son indice de calibre est de $86,2 \pm 10 \mathrm{~mm}$.

Le fruit de la mangue "Boko", de masse moyenne $390 \mathrm{~g}$, est fondamentalement constitué de la pulpe ( $67 \%$ de la masse totale) (Tableau 2).

La peau $(69,63 \pm 15,86$ g) et la graine $(45,86 \pm 9,29 \mathrm{~g})$ représentent les parties secondaires du fruit en masse. La masse de ce fruit, celle de ses différentes parties (peau, pulpe, graine et amande) et son volume $\left(380 \pm 83,35 \mathrm{~cm}^{3}\right)$ varient de façon significative. Le fruit mûr a une densité

$\rho=1,02 \pm 0,04 \mathrm{~g} / \mathrm{cm}^{3}$ proche de l'eau.

Tableau 1 : Caractéristiques morphologiques de la mangue "Boko"

\begin{tabular}{clllll}
\hline Paramètres & \multicolumn{5}{c}{ Paramètres morphologiques du fruit } \\
\cline { 2 - 6 } statistiques & $\begin{array}{l}\text { Longueur } \\
(\mathrm{mm})\end{array}$ & $\begin{array}{c}\text { Largeur } \\
(\mathrm{mm})\end{array}$ & $\begin{array}{c}\text { Epaisseur } \\
(\mathrm{mm})\end{array}$ & $\begin{array}{c}\text { Indice } \\
\text { calibre }\end{array}$ & $\begin{array}{c}\text { de } \\
\text { sphéricité }\end{array}$ \\
\hline $\mathrm{M} \pm \sigma$ & $107,94 \pm 20,09$ & $84,25 \pm 6,36$ & $72,27 \pm 5,35$ & $86,2 \pm 10,09$ & $0,88 \pm 0,52$ \\
\hline
\end{tabular}

$\mathrm{n}=40 \times 3$ (Résultats obtenus à partir de 3 fois 40 fruits) ; $M=$ moyenne ; $\sigma$ : écart type;

$M \pm \sigma=$ intervalle de confiance.

Tableau 2 : Valeurs massiques de différentes parties de la mangue "Boko"

\begin{tabular}{llllllllll}
\hline $\begin{array}{l}\text { Paramètres } \\
\text { statistiques }\end{array}$ & $\begin{array}{l}\mathrm{M}_{\mathrm{F}} \\
(\mathrm{g})\end{array}$ & $\begin{array}{l}\mathrm{Mec} \\
(\mathrm{g})\end{array}$ & $\begin{array}{l}\mathrm{Mpf} \\
(\mathrm{g})\end{array}$ & $\mathrm{Mgf}$ & $\begin{array}{l}\text { Maf } \\
(\mathrm{g})\end{array}$ & $\begin{array}{l}\mathrm{Pa} \\
(\%)\end{array}$ & $\begin{array}{l}\mathrm{Pp} \\
(\%)\end{array}$ & $\begin{array}{l}\mathrm{Vf} \\
\left(\mathrm{cm}^{3}\right)\end{array}$ & $\begin{array}{l}\rho \\
\left(\mathrm{g} / \mathrm{cm}^{3}\right)\end{array}$ \\
\hline $\mathrm{M} \pm \boldsymbol{a}$ & $390,38 \pm$ & $69,63 \pm$ & $263,72 \pm$ & $45,86 \pm$ & $45,86 \pm$ & $20,67 \pm$ & $67,29 \pm$ & $380 \pm$ & $1,02 \pm$ \\
& 82,46 & 15,86 & 62,03 & 9,29 & 4,56 & 3,86 & 0,89 & 83,35 & 0,04
\end{tabular}

$\mathrm{n}=40 \times 3$ (Résultats obtenus à partir de 3 fois 40 fruits)

$M$ : moyenne ; $\sigma$ : écart-type;

$M_{F}=$ masse de fruit $; M e c=$ masse de la peau ; Mpf= masse de la pulpe fraîche ;

$M g f=$ masse de la graine fraîche ; Maf= masse de l'amande fraîche; $P p=$ proportion de la pulpe ;

$\mathrm{Pa}=$ proportion de l'amande $; \mathrm{V} f=$ volume de fruit $; \rho=$ masse volumique.

2. Caractérisation de la pulpe de mangue "Boko" Lorsque le fruit de la mangue est mûr, sa pulpe est caractérisée par une teneur moyenne en sucres de $14,9^{\circ}$ Brix, une teneur en cendres de $1,51 \pm 0,14 \%$ et un indice de réfraction du jus de 1,36 en moyenne (Tableau 3 ).

Tableau 3: Caractéristiques physicochimiques de la pulpe de mangue "Boko"

\begin{tabular}{lccc}
\hline $\begin{array}{l}\text { Paramètres } \\
\text { statistiques }\end{array}$ & Tc $(\%)$ & $I_{R}$ & Ts $\left({ }^{\circ}\right.$ Brix $)$ \\
\hline$M \pm \sigma$ & $1,51 \pm 0,14$ & $1,36 \pm 0,004$ & $14,9 \pm 2,5$ \\
\hline$n=40 \times 3$ (Résultats obtenus à partir de 3 fois 40 fruits)
\end{tabular}

$\mathrm{M}$ : moyenne ;

$\sigma$ : écart-type;

Tc : teneur en cendres de la pulpe (\%);

$I_{R}:$ Indice de réfraction;

Ts : teneur en sucres de la pulpe ( ${ }^{\circ}$ Brix).

3. Caractérisation physicochimique du jus de pulpe de mangue "Boko"

Les échantillons de jus de mangue "Boko" provenant de trois lots de fruits de différents degrés de maturation ont été testés (Tableau 4). Le $\mathrm{pH}$, l'indice de maturation $\beta$, la teneur en sucres Tes et le taux de matière sèche Tms sont relativement plus élevés lorsque la mangue est plus mûre $(T e s=16,7 \%$ et $\operatorname{Tms}=18,8 \%$ en moyenne pour le lot III) par rapport à la mangue 
moins mûre (Tes $=13,3 \%$ et $T m s=16 \%$ en moyenne pour le lot I). Par contre, la présence d'amidon (Tableau 4), la teneur en eau $T e$, le taux de cendres $T c$ et l'acidité titrable $A T$ diminuent avec l'augmentation du degré de maturation de la mangue (diminution de $T e$ de $83,98 \%$ à $81,18 \%$, du Tc de $1,6 \%$ à $1,31 \%$ et de l' $A T$ de $7,3 \mathrm{~g}$ à $2 \mathrm{~g}$ de $\mathrm{H}_{2} \mathrm{SO}_{4} / \mathrm{l}$ du lot I au lot III).

Les fruits ayant l'indice de maturation ( $\beta$ ) égal à 18,22 sont légèrement mûrs, ceux qui ont l'indice de maturation de 51,78 sont parfaitement mûrs et ceux dont l'indice de maturation $\beta$ est égal à 83,5 sont surmaturées.

Tableau 4 : Caractéristiques physicochimiques du jus extrait de la pulpe de mangue "Boko" en fonction de la gamme de sucres ; les essais ont été répétés 3 fois. (Valeurs moyennes pour chaque lot de fruits obtenus avec des échantillons de mélange de 40 fruits)

Te : teneur en eau (\%); Tms : teneur en matière sèche; Tc : teneur en cendres (\%); Tes: teneur en extrait sucré ( ${ }^{\circ}$ Brix) ;

\section{Paramètres déterminés}

\begin{tabular}{llllllllll}
\hline $\begin{array}{l}\text { Lots de } \\
\text { mangues }\end{array}$ & $\begin{array}{l}\text { Gamme } \\
\text { de sucres } \\
\text { ('Brix) }\end{array}$ & Te $(\%)$ & $\begin{array}{l}\text { Tms } \\
(\%)\end{array}$ & Tc (\%) & $\begin{array}{l}\text { Tes } \\
\left({ }^{\circ} \text { Brix }\right)\end{array}$ & $\begin{array}{l}\text { Test } \\
\text { à } \\
\text { l'iode }\end{array}$ & pH & $\begin{array}{l}\text { AT } \\
\left(\mathrm{gH}_{2} \mathrm{SO}_{4} / \mathrm{l}\right)\end{array}$ & $\beta$ \\
\hline Lot I & $10-13,5$ & 83,98 & 16 & 1,6 & 13,3 & +++ & 3,76 & 7,3 & 18,22 \\
Lot II & $14-15,5$ & 82,23 & 17,8 & 1,63 & 14,5 & ++ & 4,3 & 2,8 & 51,78 \\
Lot III & $16-18$ & 81,18 & 18,8 & 1,31 & 16,7 & + & 4,4 & 2 & 83,5 \\
VPI/III (\%) & $/$ & $(-) 3,34$ & $(+) 17,5$ & $(-) 18,12$ & $(+) 25,26$ & $/$ & $(+) 17,02$ & $(-) 72,6$ & $/$
\end{tabular}

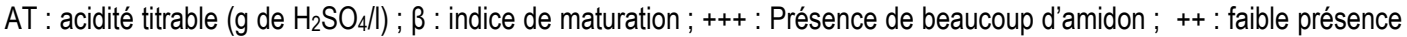
d'amidon ; + : très faible présence d'amidon; VPI/III : variation de paramètres entre lot I et lot III ; (+) : augmentation des paramètres considérés en \%; (-) : diminution des paramètres considérés en \%.

4. Transformation du jus de mangue "Boko" par fermentation éthylique contrôlée et productivité en éthanol

La consommation de sucres du moût standardisé et pasteurisé par la souche de levure Saccharomyces bayanus s'est déroulée plus ou moins rapidement après 24 heures d'incubation selon le degré de maturation de la mangue testée (Figure 1).

Elle a été plus accélérée pour le jus de mangue parfaitement mûre (avec $\beta=51,78$ ) que pour celui de la mangue surmaturée $(\beta=83,5)$. La fin de la consommation de sucres a été révélée par la stabilisation des valeurs de la teneur en sucres à partir d'un certain moment de la conduite de la fermentation (sucres résiduels $=6.7^{\circ}$ Brix à partir de 120 heures pour la mangue maturée).

L'acidité totale du jus de la pulpe de mangue a augmenté au cours de la fermentation jusqu'à une valeur maximale en rapport avec le degré de maturation de l'échantillon de mangue testée (Figure 2). Elle a été maximale après 48 heures de fermentation pour la mangue parfaitement mûre $\left(A T=4,45 \mathrm{~g}\right.$ de $\left.\mathrm{H}_{2} \mathrm{SO}_{4} / \mathrm{l}\right)$ et après 72 heures pour la mangue légèrement mûre $(A T=8,98 \mathrm{~g}$ de $\mathrm{H}_{2} \mathrm{SO}_{4} / \mathrm{l}$ ).

L'influence du degré de maturation de la mangue "Boko" sur le rendement de la fermentation et la productivité en éthanol a été testée (Figure 3 ). Dans les trois cas de degré de murissement de la mangue, le rendement de consommation ou transformation de sucre Rt par la levure a été comparable $(65,25 \%, 65,453 \%$ et $65,68 \%$ de sucre consommé dans le cas respectivement de mangue légèrement mûre, mûre et surmaturée). Par contre, le rendement de conversion de sucre en éthanol $R c$ et la productivité totale en éthanol $P_{T E}$ ont varié en fonction du degré de mûrissement du fruit. Pour le jus de mangue parfaitement mûre (avec $\beta=51,78$ ), le rendement de conversion de sucre en éthanol $R c$ et la productivité totale horaire en éthanol $P_{T E}$ ont été maximum $\left(R c=88,75 \%\right.$ et $P_{T E}=0,61 \mathrm{~g}$ d'éthanol formé par $\mathrm{kg}$ de pulpe et par heure pour $\beta$ $=51,78$ ). Mais, ces deux paramètres de fermentation ont été plus faibles dans les deux cas extrêmes du mûrissement du fruit $(R c=$ 


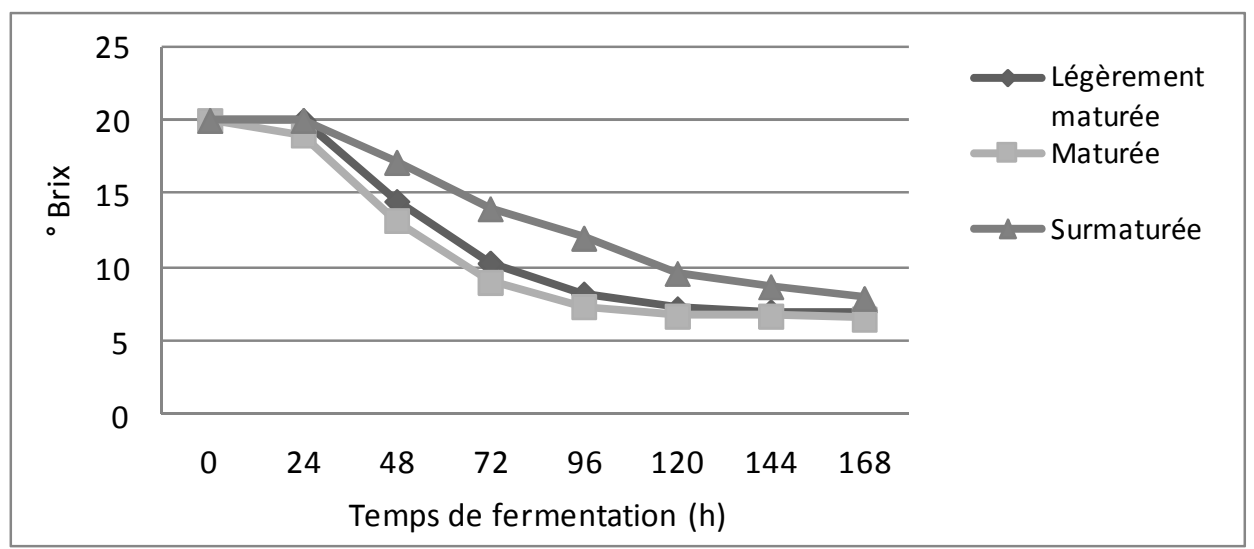

Figure 1 : Evolution de l'extrait sucré (en \%) au cours de la fermentation éthylique en fonction du degré de maturation u fruit (mangue légèrement maturée, maturée et surmaturée)

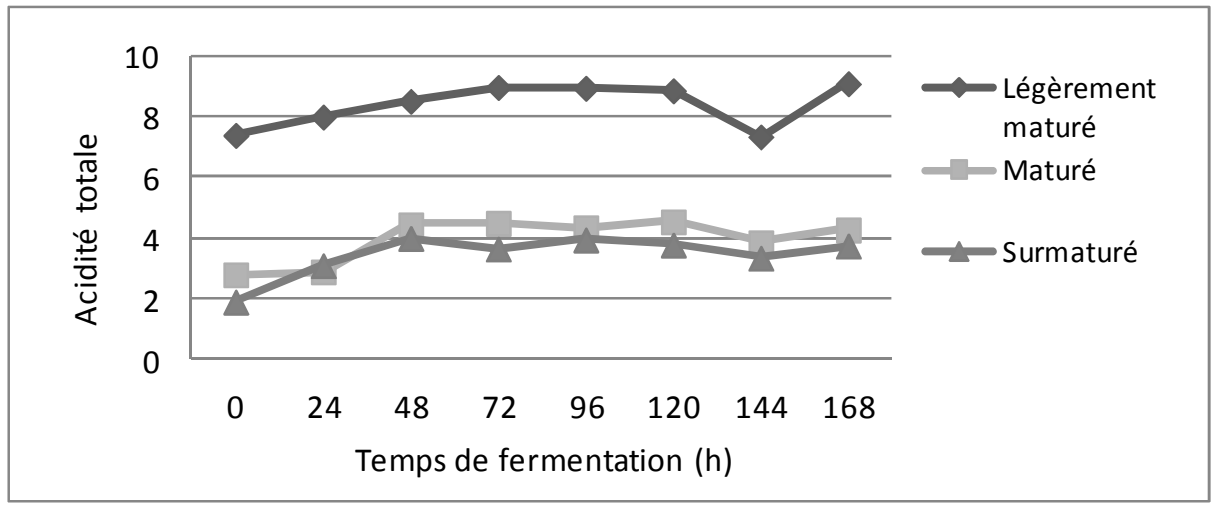

Figure 2 : Evolution de l'acidité totale (en $\mathrm{g} \mathrm{de} \mathrm{H}_{2} \mathrm{SO}_{4} /$ ) au cours de la fermentation éthylique en fonction du degré de maturation du fruit.

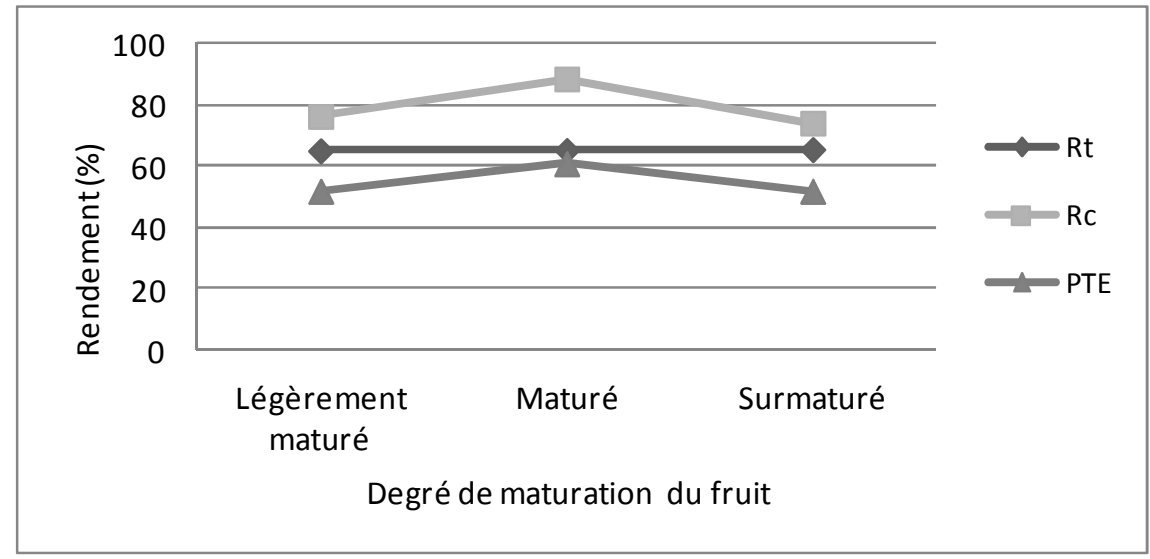

Figure 3 : Relation entre degré de maturation du fruit de la mangue et rendements de consommation, de conversion de sucres et productivité totale en éthanol.

$R t$ : rendement de consommation de sucres (\%); Rc: rendement de conversion de sucre en éthanol (\%); PTE: productivité totale horaire en éthanol (en $10^{-2} \mathrm{~g}$ d'éthanol formé/kg de pulpe/h). 


\section{DISCUSSION}

La mangue "Boko" présente des caractéristiques morphologiques proches de celles observées sur les variétés de mangue cambodiana et faisanzon introduites au Centre M'vuazi à Kinshasa [1]. La détermination des paramètres physiques de la mangue Boko pourrait aider à la conception d'équipements relatifs à la mécanisation des opérations de traitement de fruits pour la fermentation, notamment le lavage, le calibrage, l'épluchage, le dénoyautage, le broyage de la pulpe et l'extraction du jus. La densité de la mangue Boko $\left(\rho=1,02 \pm 0,04 \mathrm{~g} / \mathrm{cm}^{3}\right)$, proche de celle de l'eau pourrait permettre la flottaison des fruits et faciliter leur nettoyage en cuve à grande échelle. Sa masse moyenne $(390 \mathrm{~g})$ est très proche des variétés lippens et broagt $(400 \mathrm{~g}$ en moyenne) [5], et amélie (370g) [22]. Son rendement élevé en pulpe ( $67 \%$ de pulpe) serait plus bénéfique pour la transformation du jus extrait en éthanol par rapport à celui des variétés lippens, broagt, kent et amélie (30-50\% de pulpe) [5].

Les mesures de divers paramètres physicochimiques des échantillons de la pulpe de mangue "Boko" testée varient en fonction du degré de murissement physiologique de ce fruit. Le $\mathrm{pH}$, le taux de matière sèche et la teneur en sucres augmentent lorsque la mangue "Boko" est de plus en plus mûre. A la surmaturation, la teneur en sucres atteint une valeur très élevée $\left(19^{\circ}\right.$ Brix) suite à la dessiccation saisonnière $\left(33^{\circ} \mathrm{C}\right.$ en moyenne) subie par cette mangue comme pour les autres fruits tels que le raisin [8].

Par contre, un phénomène inverse a été révélé, notamment pour la teneur en eau, en cendres et le degré d'acidité au cours du mûrissement de la mangue "Boko" (Tableau 4). Les mangues moins mûres sont plus riches en eau ( $83,98 \%$ d'eau) que les mangues plus mûres $(81,18 \%$ d'eau pour la mangue surmaturée). Cette tendance, contraire à celles citées par Badara $\mathrm{Ba}$ [23], confirme les résultats de De Laroussilhe [1] qui note une teneur moyenne en eau de $90 \%$ pour la mangue verte et $86,3 \%$ pour la mangue mûre. Le degré d'acidité diminue significativement de $72,6 \%$ lorsque la mangue passe du stade de fruit légèrement mûr à la surmaturation [3; 23].

Pour la transformation technologique du jus de la pulpe de mangue "Boko" par voie fermentaire éthylique, le choix du stade de maturation physiologique du fruit doit être basé sur la couleur de peau et l'indice de maturation $\beta$ comme c'est le cas dans la fabrication du vin de raisin [8; 24]. L'acidité de la pulpe de la mangue "Boko" mûre doit être égale ou supérieure à $2,8 \mathrm{~g}$ de $\mathrm{H}_{2} \mathrm{SO}_{4} / \mathrm{l}$ afin d'éviter une fermentation éthylique incomplète notée dans le cas du jus de la pulpe de mangue surmaturée ayant une acidité totale très faible.

Pour remédier à une telle situation de faible acidité de la mangue surmaturée, une acidification indirecte serait nécessaire en $y$ apportant de la pulpe de mangue légèrement mûre afin d'augmenter l'acidité à environ 2,8 à $3 \mathrm{~g}$ de $\mathrm{H}_{2} \mathrm{SO}_{4} / l[8]$.

\section{CONCLUSION}

En mûrissant, la teneur en extrait sucré, le taux de matière sèche et le $\mathrm{pH}$ de la pulpe de mangue "Boko" augmentent, mais la teneur en eau, en cendres et l'acidité totale décroissent.

Le degré de mûrissement de la mangue "Boko" peut être évalué sur la base de couleur de la peau et d'indice de maturation $\beta$ (rapport entre teneur en sucres et acidité totale).

La mangue "Boko ", grâce à son jus très sucré extrait de la pulpe, est une bonne matière première adéquate pour la transformation technologique par voie fermentaire éthylique. Le rendement de transformation des sucres totaux en éthanol et la productivité horaire en éthanol sont meilleures lorsque l'indice de maturation $\beta$ de la mangue est de $\beta=51,78$. Au cours de la fermentation éthylique du jus de mangue, les valeurs maximales de ces paramètres technologiques sont obtenues avec une valeur d'indice de maturation intermédiaire $\beta=51,78$ considérée comme valeur optimale du degré de maturation de l'échantillon de mangue "Boko" étudiée.

\section{BIBLIOGRAPHIE}

1. de Laroussilhe F. 1980. Le manguier. Maisonneuve et Larose, Paris. Collection Techniques agricoles et productions tropicales 29.

2. Martine F. 2003.Transformer les fruits tropicaux. Collection Le point sur les technologies. Dumas-Titoulets Imprimeurs. 
3. Pamploma G.R. 2007. Santé par les aliments. Collection "Nouveau style de vie": Editorial Safeliz, S.L, 1ère Edition.

4. Ameyapoh Y., Wokpor K, de Souza, C. 2006. Identification et sélection de souches de levure performantes pour la production d'alcool. Journal des Sciences 6(1): 30-40.

5 Anonyme 2 1997. La mangue séchée. Des fruits choisis avec soin avant transformation. Bulletin du réseau TPA, s/c CRET, 14: 55-56.

6 Silou T.1996. Le safoutier (Dacryodes edulis), un arbre mal connu. Fruits 51: 47-60.

7 C.E.E 1973. Directives des analyses; Analyse des composants des aliments des animaux. Méthodes décrites dans les Annexes des Directives, No71/199 C.E.E du 15.6.77; $\mathrm{N}^{\circ}$ 71/393 C.E.E. du 18.11.71; No 72/199 C.E.E du 27.4.72; $\mathrm{N}^{\circ}$ 73/46 C.E.E du 5.12.72. Institut d'Enseignement Supérieur Agricole de l'Etat à Huy, Belgique.

8 Navarre J.P., Navarre C. 1986. Manuel d'œnologie. Ed. J.B. Baillière. $4^{\mathrm{e}}$ Ed. Paris. 287p.

9 Oyeyiola G.P. 1991. Fermentation of millet to produce kamu a Nigeria starch-cake food. World Journal of Microbiology and Biotechnology 7: 196-201.

10 Diakabana P., Louembé D. and Kobawila S.C. 2007. Physico-chimical and Biochemical characteristics of the fermentation of boganda, a brandy of Congo. Research Journal of Biotechnology 2 (1): 18-25.

11 Diakabana P., Nyanga-Koumou C.A.M., Kobawila SC, Louembé D and Derdelinckx G. 2008. Study of characteristic parameters of traditional process in the production of " boganda", a brandy of Congo. Int.J.Biol.Chem.Sci. 2 (3): 258- 271.

12 Gauthier M., Tremblay R et Deslauriers M. 2005. Contrôle du procédé de fermentation alcoolique. Institut de Technologie Agroalimentaire, Québec.

13 Lonvaud-Funel A., Masclef J.P.h., Joyeux A. et Paraskevopoulos Y. 1988. Etude des interactions entre levures et bactéries lactiques dans le moût de raisin. Connaissance Vigne Vin 22(1): 11-24.
14 Soufleros E., Bertrand E. 1979. Rôle de la souche de levure dans la production des substances volatiles au cours de la fermentation du jus de raisin. Connaissance Vigne Vin 13 (3): 185-189.

15 Marc I. 1982. La levure en fermentation: Etude bibliographique. Bios 13(10): 45-53.

16 De Clerck J. 1963. Cours de Brasserie. Université Catholique de Louvain, Editieur: Chaire Jean De Clerck. Tome 2, ॥ème Ed., Belgique. 953p

17 E.B.C. 1987. Analytica-EBC.

18 Leveau J.V., Bouix M. 1988. Cinétiques microbiennes. In: Biotechnologie, Scriban R., coordonnateur. Technique et Documentation Lavoisier, 3ème Ed. Paris : 203-232.

19 Engasser J.M. 1988. Modélisation du processus de fermentation .In : Biotechnologie, Scriban R, coordonnateur. Techn. Doc. Lavoisier, 3ème Ed. Paris : 301-323.

20 Diakabana P. 2006. Optimisation du procédé traditionnel de production de boganda, une eaude-vie du Congo. Thèse de Doctorat unique, Faculté des Sciences, Université Marien Ngouabi, Brazzaville, Congo.145 p.

21 Larrieu J. 1988. Utilisation de la statistique en gestion de la qualité. In: Biotechnologie, Scriban R, coordonnateur. Technique et Documentation Lavoisier. 3ème Ed. Paris : 301679.

22 Kameni A., Yézouma C., Dandjouma A., Chawhi A., Namata A., Lenga J-B., Desmorieux H. 2005. Séchage de la mangue : Comparaison des divers procédés et variétés. In : Maîtrise des procédés en vue d'améliorer la qualité et la sécurité des aliments. Utilisation des O.G.M. Analyse des risques en agroalimentaire. Ouagadougou, 8-11 Novembre 2005.

23 Badara A. 1992. Technologie de transformation des produits alimentaires de base en Afrique. Session d'échange de Dakar, Sénégal.

24 Duteurtre 1986. La science du champagne. La recherche 183 : 1475-1489. 